\title{
APUAMA: A Software tool for reaction rate calculations
}

\author{
Euclides, H. O. ${ }^{1}$, Barreto, P. R. P. ${ }^{1}$ \\ ${ }^{1}$ Instituto Nacional de Pesquisas Espaciais, São José dos Campos, SP, Brasil \\ Aluno de Mestrado do curso de Ciência e Tecnologia de Materiais e Sensores - CMS. \\ henriqueuclides@gmail.com
}

\begin{abstract}
APUAMA is a free software designed to determine the reaction rate and thermodynamic properties of chemical species of a reagent system. With data from electronic structure calculations, the APUAMA determine the rate constant with tunneling correction, such as Wigner, Eckart and small curvature, and also, include the rovibrational level of diatomic molecules. The results are presented in the form of Arrhenius form, for the reaction rate, and the thermodynamic properties are written down in the polynomial form. The word APUAMA means "fast" in Tupi-Guarani Brazilian language, then the code calculates the reaction rate on a simple, intuitive graphic interface, the form fast and practical. As program output, there are several text files with tabulated information for rate constant, rovibrational levels, energy barriers and enthalpy of reaction, Arrhenius coefficient, and also, the option to the User save all graphics in BMP format.
\end{abstract}

Keywords APUAMA code; Rate constant; Quantum chemistry; Rovibrational Levels; Thermodynamic properties.

\section{Introduction}

Knowledge of the thermodynamic properties of chemical species and the rate with all the species reacts is of fundamental importance for studies such as combustion processes; the atmosphere for greenhouse effect, acid rain, ozone layer, pollution, interstellar space compositions; gas phase during the growth of CVD (chemical vapour deposition) thin films and others applications. In all these applications, the acknowledgement of the constant rate is essential to know the speed with which they occur, or the change of the concentrations of the reagents and products as function of time, that is related with the rate constant and temperature. With this idea in mind Barreto et. al. [Barreto et al. 2003] developed a very simple code, based in transition state theory [Truhlar et al. 1985], to calculate the rate constant with only the Wigner tunneling correction [Wigner 1932]. Posterioly, this code was improved [Barreto et al. 2005] to include the minimum energy path (MEP) and the Eckart tunneling correction [Gonzalez-Lafont et al. 1991]. The first two version [Barreto et al. 2003, Barreto et al. 2005] were written in Fortran programming language, while the APUAMA is written in $\mathrm{C}++$ programming language.

For all calcutation, it is necessary to know the geometries, energies and vibrational frequencies of reactants, products and saddle point (transition structure), which are previously obtained via electronic structure calculations, using specific programs for this purpose, such as Molpro [Werner et al. 2010], Gaussian [Frisch et al. 2003] Gamess [M.W.Schmidt et al. 1993], Molcas [Andersson et al. 2002] and others. The quality of 
the final results will be related with the quality of the input data from electronic structure calculations.

The paper is organized as follows, in Section 2.1 are given the theory behind the APUAMA, while the main features of the program are presented and discussed in Section 2.2, some examples are presented and discused in Section 3 and conclusions are following in Section 4.

\section{Metodology}

\subsection{TST - Transition state theory}

APUAMA was developed using the transition structure theory (TST) [Truhlar et al. 1985], where it is assumed to have a structure, not stable, with minimum energy between reactant and products, and we have a treatment more statistical than collisional for the reaction.

Considering the bimolecular reaction between $\mathrm{A}$ and $\mathrm{BC}$, where $\mathrm{A}$ and $\mathrm{BC}$ could be atoms or molecules given by:

$$
\mathrm{A}+\mathrm{BC} \Longrightarrow \mathrm{X}^{\ddagger} \Longrightarrow \mathrm{AB}+\mathrm{C}
$$

We define an intermediate structure, in a condition of "nearly balanced" minimum energy between reagents and products, which is usually called transition structure. The potential energy $V$ of a reaction system depends on the relative positions of $\mathrm{A}, \mathrm{B}$ and $\mathrm{C}$, the interatomic distances specified $R_{\mathrm{AB}}, R_{\mathrm{BC}}$ and interatomic angle $\theta_{\mathrm{ABC}}$. For large values of $R_{\mathrm{BC}}$, there is no interaction between $\mathrm{A}$ and $\mathrm{BC}$, and the cross section in this region corresponds to the potential energy curve for $\mathrm{BC}$ molecule, the initial state, while for large value of $R_{\mathrm{AB}}$ corresponds the final state, $\mathrm{AB}+\mathrm{C}$, and large value of $R_{\mathrm{BC}}$ and $R_{\mathrm{AB}}$ corresponds the completed dissociated state, $\mathrm{A}+\mathrm{B}+\mathrm{C}$, as shown in the figure 1 .

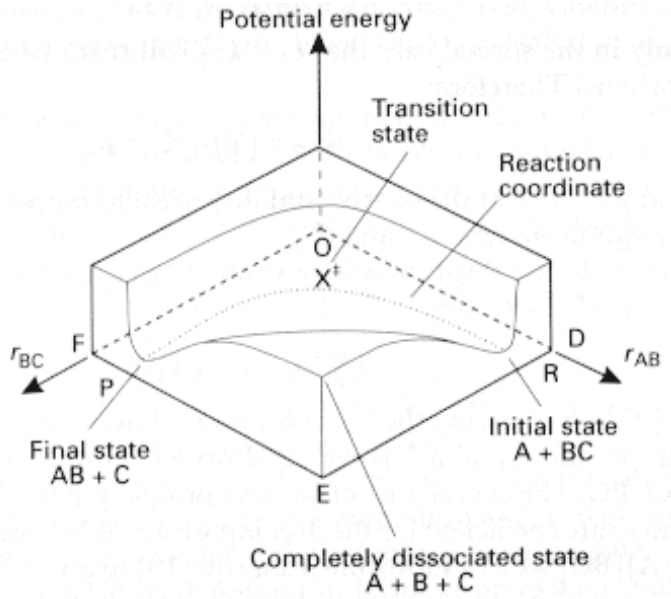

Figura 1. 3D potential energy surface for a collinear reaction $A+B C=A B+C$

The equation for TST is:

$$
k(T)=\frac{k_{B} T}{h} \frac{Q_{X^{\ddagger}}}{Q_{A} Q_{B C}} \exp \left(-\frac{V_{a}^{G^{\ddagger}}}{R T}\right)
$$


Where $k_{B}$ is the Boltzmann's constant, $h$ Planck's constant, $T$ temperature, $Q_{X^{\ddagger}}$, $Q_{\mathrm{A}}$ and $Q_{\mathrm{BC}}$ are the partition function of TS (transition structure), reactants A and $\mathrm{BC}$, respective, $V_{a}^{G^{\ddagger}}$ energy barrier with the zero point energy correction. The partition function for all species is given by the product of the partition function of translation $\left(Q_{\text {trans }}\right)$, rotation $\left(Q_{r o t}\right)$, vibration $\left(Q_{v i b}\right)$ and electronic $\left(Q_{\text {elet }}\right)$ as function of temperature and are given by Table 1 . The translation partition function $\left(Q_{\text {trans }}\right)$ depends only of the mass of the system $m$, has three degree of freedom, it is the only partition function that has unit $\left(\mathrm{m}^{3}\right)$ and order of magnitude around $10^{33}$. The rotational partition function has two degree of freedom for linear molecule, and three degree of freedom for nonlinear molecule and is dimensionless, it depends of the moment of inertia $I$ or product of inertia $I_{m}$ for linear or nonlinear molecule, respectively, and, also, the external symmetry $\sigma$, and it is around $1-10^{3}$ order of magnitude. The vibrational partition function $\left(Q_{v i b}\right)$ has $l=3 N-5$ or $l=3 N-6$ degrees of freedom for linear molecule and nonlinear molecule, respectively, where $N$ is the number of atoms in the molecules, it depends of the vibrational frequencies $\nu_{i}$ and degeneracy $g_{i}$, the order of magnitude depends of the number of degree of freedom $(l)$ and is around $1-10^{l}$. The electronic partition function depends on the electronic energy level $\varepsilon_{i}$ and degeneracy $g_{i}$. The transition structure has one less vibrational degree of freedom, corresponding to the reaction coordinate, in this way, the number of vibrational degrees of freedom will be $l=3 N-6$ or $l=3 N-7$ for linear and nonlinear molecules, respectively.

Tabela 1. Partition function for the reactants, products and TS, when $Q=$ $Q_{\text {trans }} Q_{\text {rot }} Q_{v i b} Q_{\text {elet }}$

\begin{tabular}{|c|c|c|c|}
\hline$Q$ & Degree of Freedom & Partition Function & Order of Magnitude \\
\hline Translation & 3 & $Q_{\text {trans }}=\left(\frac{2 \pi m k_{B} T}{h^{2}}\right)^{3 / 2}$ & $10^{33}$ \\
\hline Rotation 2D & 2 & $Q_{r o t-2 D}=\frac{8 \pi^{2} I k_{B} T}{\sigma h^{2}}$ & $10-10^{2}$ \\
\hline Rotation 3D & 3 & $Q_{r o t-3 D}=\frac{\sqrt{\pi}}{\sigma}\left(\frac{8 \pi^{2} I_{m} k_{B} T}{h^{2}}\right)^{3 / 2}$ & $10^{2}-10^{3}$ \\
\hline Vibration & $\begin{array}{l}l=3 N-5 \\
l=3 N-6\end{array}$ & $Q_{v i b}=\prod_{i=1}^{l}\left[1-\exp \left(\frac{-h c \nu_{i}}{k_{B} T}\right)\right]^{g_{i}}$ & $1-10^{l}$ \\
\hline Electronic & - & $Q_{\text {elet }}=\sum_{i=0}^{n} g_{i} \exp \left(\frac{-\varepsilon_{i}}{k_{B} T}\right)$ & 1 \\
\hline \multicolumn{4}{|c|}{$\begin{array}{l}T \text { is temperature, } h \text { Planck's constant, } k_{b} \text { Boltzmann's constant, } m \text { is the system mass, } \\
I \text { inertial moment for linear molecules, } I_{m} \text { inertial product for non linear molecules, } \\
c \text { speed of light, } \nu_{i} \text { vibrational modes, } g_{i} \text { degeneracy } \varepsilon_{i} \text { electronic energy. }\end{array}$} \\
\hline
\end{tabular}

\subsection{Features of APUAMA}

APUAMA was developed with the purpose of calculating the reaction rate in a fast and intuitive way, being a small and optimized program that can be executed in machines of low power, offering support for computers with operating systems such as Linux or Windows. 
Our program has a simple graphic interface with several buttons/menus, they can give access to read the input data, run the code, write the output data or salve the figures generated. One of the buttons is an example how to write the input files for reactant/products, transition state, reaction and the potential energy surface of diatomic molecule, this button is called "Input Type", as shown in the figure 2. In this figure is, also, possible to see all the buttons for input data, and menus for calculations.

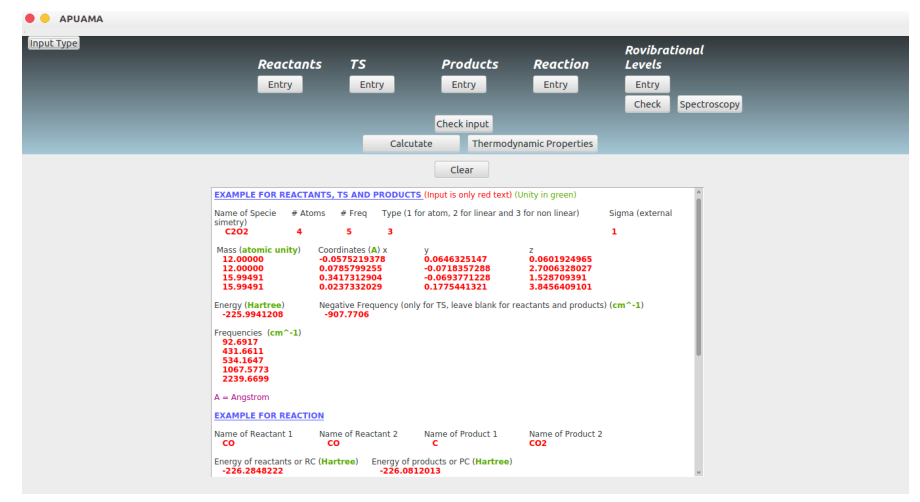

Figura 2. APUAMA screen for button "Input type", with a example of all kind of inputs

After entering with the data for reagents, TS, products and reaction, the program will be able to calculate the rate and the thermodynamic properties. The data will be, automatically, displayed on the screen, as seen in figure 3. It shows the reaction $\mathrm{Cl}+\mathrm{HF}=$ $\mathrm{H}+\mathrm{FCl}$, the first block defines the reactants, the second one the TS, the third one the product, in each one is defined the species, number of atoms, number of frequencies, type of molecule (atomic species, linear or nonlinear molecule), external symmetry and energy, and the last block shows the reaction path, which is the order of the species and the total energy of the system. During the calculation this screen can be display again by pressing the button "Check Input".

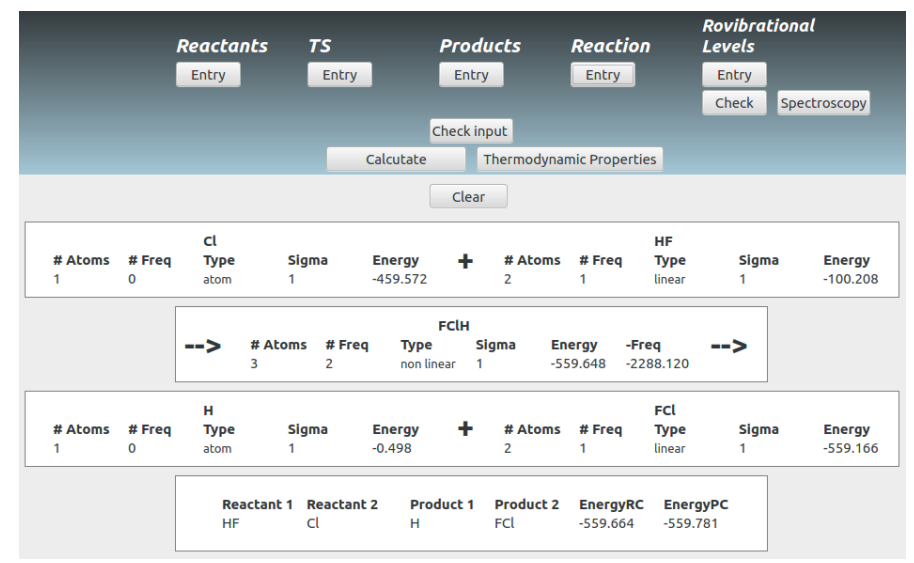

Figura 3. Screen example of a reaction $\mathrm{Cl}+\mathrm{HF}=\mathrm{H}+\mathrm{FCl}$ with all the necessary data

The rate constant and thermodynamic properties is calculated in the "Calculate" or "Thermodynamic Properties" menu, respectively. If the input files are correct, a folder 
with the name of the TS will be created and the output data and/or figures will be saved in this new directory. In the case of rate constant, there is four kind of results, rate constant, MEP, energies (barriers, reaction enthalpies and skew angles) and modificed Arrhenius [Kooij 1893], as shown in figure 4(a). The rate constant is displayed in figure 4(b), with all the tunnelling corrections. The figure could be saved as image file and the data is save in a ASCII file, that could be used in other programs.

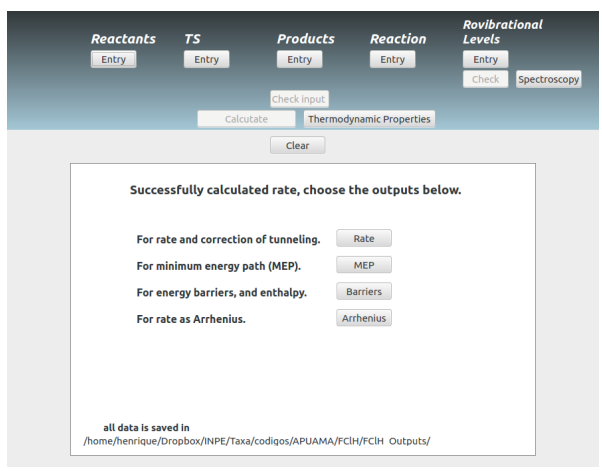

(a) Calculation menu

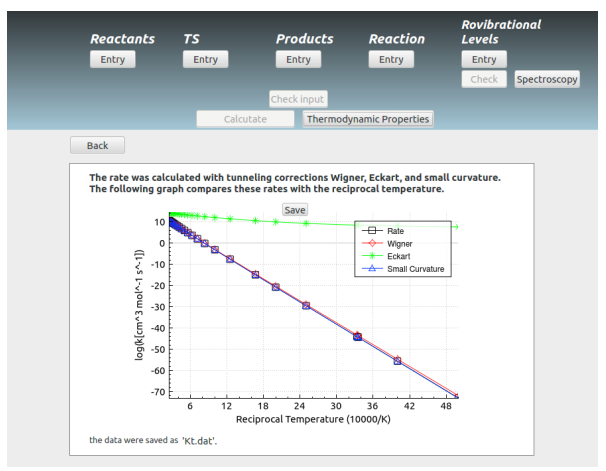

(b) Rate calculation results

Figura 4. Calculation in APUAMA

With appropriated data of reagents or products, when necessary, the rovibrational levels [Dunham 1932] and spectroscopy properties can be determined and used to calculated the rate constant. For this we enter with the values of the potential energy curve, previously calculated and insert the level $\nu$ for the vibration and $J$ for rotation.

\section{Results and Discussions}

To test the APUAMA, we calculated the reaction rate calculations for several systems, and when possible, compared then with experimental data. Up to now, we have test it for unimolecular and bimolecular reaction with systems up to twelve atoms, but there is no limit for the number of atoms we can use. To exemplify the use of the program here, we calculate the reaction $\mathrm{HF}+\mathrm{Cl}=\mathrm{H}+\mathrm{FCl}$ where we can use the rovibrational level for reactant and products.

The reactant, product and TS for this reaction were optimized using Gaussian09 [Frisch et al. 2003] code in MP2/aug-cc-pVTZ method and the energy were determined at CCSD(T)/aug-cc-pVQZ. The geometry parameters and frequencies for this system will not be presented here, and the frequency correction factor used was 0.997630 .

The thermodynamic properties were calculated and presented in figure 5, that compares the enthalpy and entropy for $\mathrm{H}, \mathrm{Cl}, \mathrm{HF}$ and $\mathrm{FCl}$. 

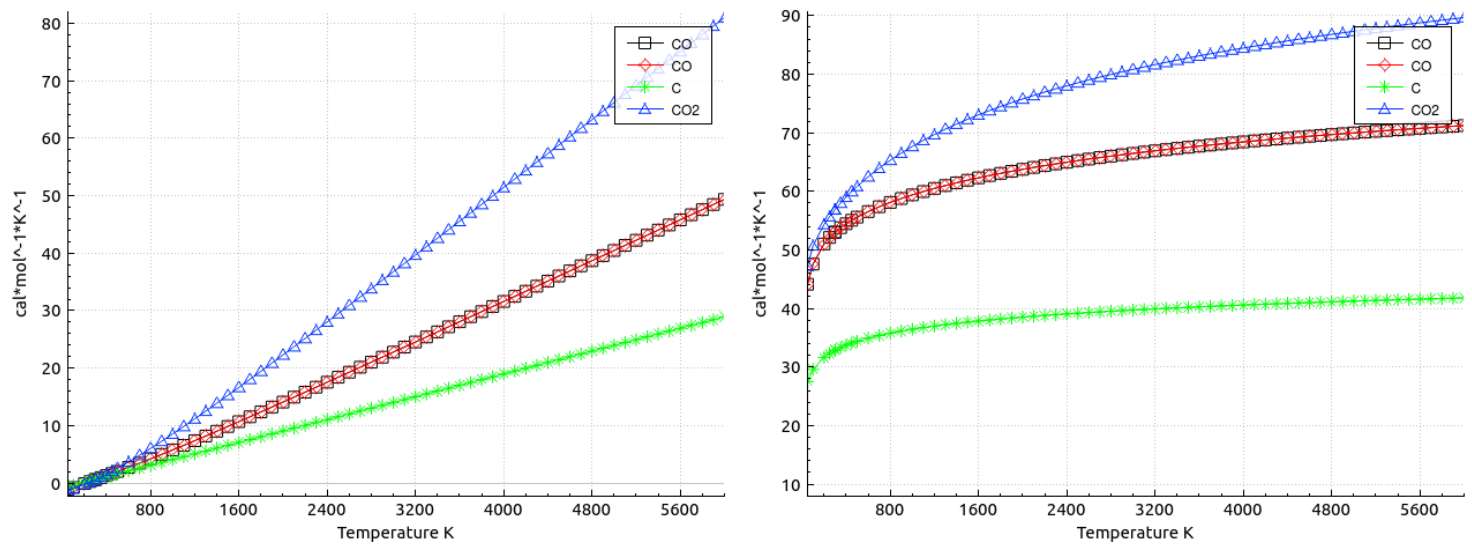

Figura 5. (a) Enthalpy for $\mathrm{HF}+\mathrm{Cl}=\mathrm{H}+\mathrm{FCl}$, (b) Entropy for $\mathrm{HF}+\mathrm{Cl}=\mathrm{H}+\mathrm{FCl}$

This reaction has a heat of formation of $68.34 \mathrm{kcal} \mathrm{mol}^{-1}$, with barrier in the forward direction of $79.27 \mathrm{kcal} \mathrm{mol}^{-1}$ and in the reverse direction of $10.80 \mathrm{kcal} \mathrm{mol}^{-1}$. A level $(4,0)$ in the reactant means a reduction of $1.16 \mathrm{kcal} \mathrm{mol}^{-1}$ in the forward direction barrier, while a level of $(5,5)$ in the products means a reduction of $1.62 \mathrm{kcal} \mathrm{mol}^{-1}$ in the reverse direction barrier, because the rovibrational energy of HF is much higher than the $\mathrm{FCl}$, so lower pair of $(\nu, J)$ could give higher energy.

We calculate the rate with rovibrational level $(5,5)$ for the product $F C l$, but since the barrier in the reverse direction is only $10 \mathrm{kcal} \mathrm{mol}^{-1}$, it is not necessary to include this rovibrational level even reducing it by $2 \mathrm{kcal} \mathrm{mol}^{-1}$. For the reagent $H F$, where the barrier in the direct direction is $80 \mathrm{kcal} \mathrm{mol}^{-1}$, we can include the level $(4,0)$ and obtain a reduction of $4 \mathrm{kcal} \mathrm{mol}^{-1}$. The figure $6 \mathrm{a}$ shows the rate with the inclusion of these levels compared to the tunnelling corrections, and the figure $6 \mathrm{~b}$ shows the MEP with the zero point correction.
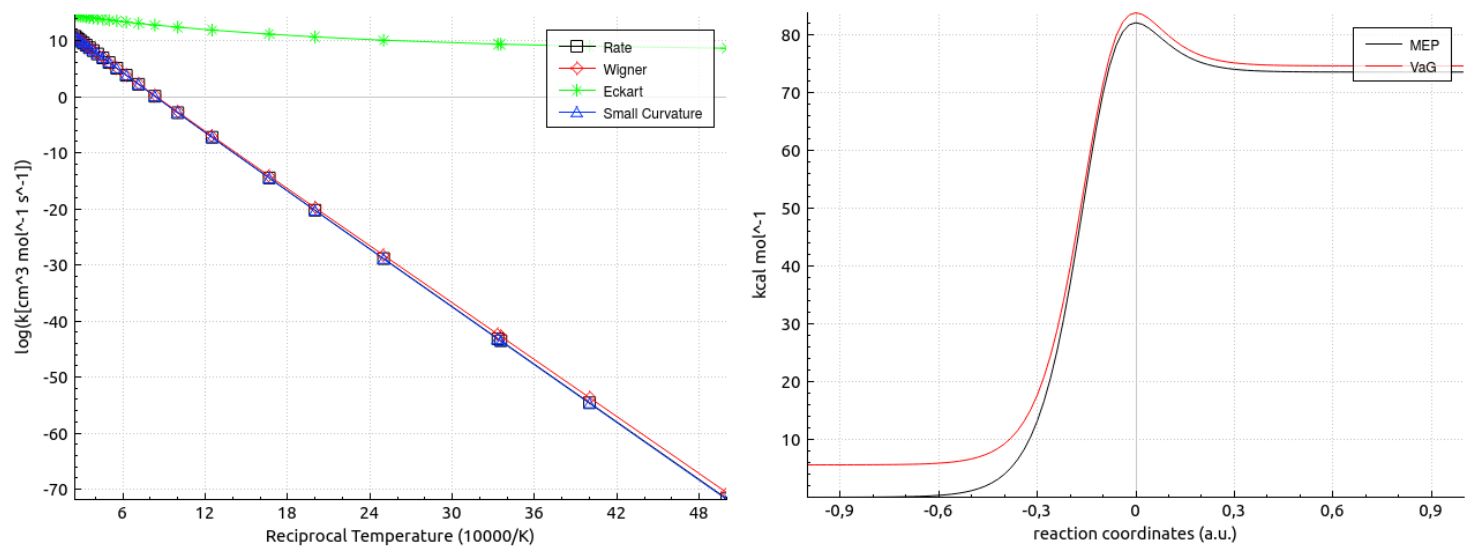

Figura 6. (a) Reaction rate for $H F+C l=H+F C l$ with $(5,5)$ rovibrational levels on the $F C l$ and $(4,0)$ in $H F$, (b) MEP for $H F+C l=H+F C l$ with the same rovibrational levels 


\section{Conclusion}

Our goal with APUAMA was to develop a simple self-explanatory, easy-to-use graphical interface code and quick results for the determination of the reaction rate for general systems and to calculated thermodynamic properties. The developed code allows us to calculate the rate of bimolecular and unimolecular systems, taking into account rovibrational levels of diatomic species, since the potential energy curve is specified a priori. The code presented was tested for small systems 3 and 4 atoms, taking into account the rovibration levels and large systems up to 12 atoms, without limiting the number of atoms. It is easy and fast to use, does not need a very elaborated manual and could run on Windows or Linux computer. The code will be available free of charge upon user request.

Acknowledgements: This research was funded by the CAPES.

\section{Referências}

Andersson, K., Barysz, M., Bernhardsson, A., Blomberg, M. R. A., Cooper, D. L., Fülscher, M. P., de Graaf, C., Hess, B. A., Karlström, G., Lindh, R., Malmqvist, P.-A., Nakajima, T., Neogrády, P., Olsen, J., Roos, B. O., Schimmelpfennig, B., Schütz, M., Seijo, L., Serrano-Andrés, L., Siegbahn, P. E. M., Stalring, J., Thorsteinsson, T., Veryazov, V., and Widmark, P.-O. (2002). MOLCAS Version 5.4. Lund University, Sweden.

Barreto, P., Vilela, A., and Gargano, R. (2003). J. Mol. Struct. - Theochem, 639:167.

Barreto, P., Vilela, A., and Gargano, R. (2005). Int. J. Quantum Chem., 103:685.

Dunham, J. L. (1932). The weltzel-brillouin-kramers method of solving the wave equation. Phys. Rev., 41:713.

Frisch, M. J., Trucks, G. W., Schlegel, H. B., Scuseria, G. E., Robb, M. A., Cheeseman, J. R., , J. A Montgomery, J., Vreven, T., Kudin, K. N., J. C. Burant, J. M. M., Iyengar, S. S., Tomasi, J., Barone, V., Mennucci, B., M. Cossi, G. S., Rega, N., Petersson, G. A., Nakatsuji, H., Hada, M., Ehara, M., Toyota, K., Fukuda, R., Hasegawa, J., Ishida, M., Nakajima, T., Honda, Y., Kitao, O., Nakai, H., Klene, M., Li, X., Knox, J. E., Hratchian, H. P., Cross, J. B., Adamo, C., Jaramillo, J., Gomperts, R., Stratmann, R. E., Yazyev, O., Austin, A. J., Cammi, R., Pomelli, C., Ochterski, J. W., Ayala, P. Y., Morokuma, K., Voth, G. A., Salvador, P., Dannenberg, J. J., Zakrzewski, V. G., Dapprich, S., Daniels, A. D., Strain, M. C., Farkas, O., Malick, D. K., Rabuck, A. D., Raghavachari, K., Foresman, J. B., Ortiz, J. V., Cui, Q., Baboul, A. G., Clifford, S., Cioslowski, J., Stefanov, B. B., Liu, G., Liashenko, A., Piskorz, P., Komaromi, I., Martin, R. L., Fox, D. J., Keith, T., Al-Laham, M. A., Peng, C. Y., Nanayakkara, A., Challacombe, M., Gill, P. M. W., Johnson, B., Chen, W., Wong, M. W., Gonzalez, C., and Pople, J. A. (2003). Gaussian 03, revision c.02. Technical report, Gaussian, Inc., Pittsburgh PA, gaussian.

Gonzalez-Lafont, A., Truong, T. N., and Truhlar, D. G. (1991). Interpolated variational transition- state theory: Practical methods for estimating variational transition-state properties and tunneling contributions to chemical reaction rates from electronic structure calculations. J. Chem. Phys., 95:8875.

Kooij, D. (1893). Z Phys Chem., 12:155. 
M.W.Schmidt, K.K.Baldridge, J.A.Boatz, S.T.Elbert, M.S.Gordon, J.H.Jensen, S.Koseki, N.Matsunaga, K.A.Nguyen, S.Su, T.L.Windus, M.Dupuis, and J.A.Montgomery (1993). General atomic and molecular electronic structure system. J. Comput. Chem., 14:1347-1363.

Truhlar, D. G., Isaacson, A. D., , and Garrett., B. C. (1985). Theory of Chemical Reaction Dynamics, volume 2. CRC Press, Inc., Boca Raton, Florida Oxford Science, Boca Raton, Florida.

Werner, H.-J., Knowles, P. J., Knizia, G., Manby, F. R., Schütz, M., Celani, P., Györffy, W., Kats, D., Korona, T., Lindh, R., Mitrushenkov, A., Rauhut, G., Shamasundar, K. R., Adler, T. B., Amos, R. D., Bernhardsson, A., Berning, A., Cooper, D. L., Deegan, M. J. O., Dobbyn, A. J., Eckert, F., Goll, E., Hampel, C., Hesselmann, A., Hetzer, G., Hrenar, T., Jansen, G., Köppl, C., Liu, Y., Lloyd, A. W., Mata, R. A., May, A. J., McNicholas, S. J., Meyer, W., Mura, M. E., Nicklass, A., O’Neill, D. P., Palmieri, P., Peng, D., Pflüger, K., Pitzer, R., Reiher, M., Shiozaki, T., Stoll, H., Stone, A. J., Tarroni, R., Thorsteinsson, T., and Wang, M. (2010). Molpro, version 2010.1, a package of ab initio programs. see.

Wigner, E. (1932). Z. phys. Chem., 17:353. 